\title{
The alien hand and related signs
}

\author{
Rachelle Smith Doody, Joseph Jankovic
}

\begin{abstract}
Alien limb sign includes failure to recognise ownership of one's limb when visual cues are removed, a feeling that one body part is foreign, personification of the affected body part, and autonomous activity which is perceived as outside voluntary control. Although the hand is most frequently affected, any limb or combination of limbs may fulfil the alien limb criteria. Alien hand sign should be reserved for cases in which the hand feels foreign together with observable involuntary motor activity. To characterise this phenomenon, seven patients with alien hand sign and other motor or behavioural manifestations are described. Aetiologies included multiple infarcts and cortobasal ganglionic degeneration (CBD). In this study, all patients had apraxia in response to verbal commands and problems with bimanual coordination. Most displayed non-goal directed involuntary motor activities, and two had self destructive motor behaviours. Grasp reflex occurred with alien hand due to either aetiology. Cortical reflex myoclonus was frequently seen in CBD patients. The phenomenological spectrum is reviewed, a diagnostic protocol proposed, and possible anatomical bases of alien hand discussed.
\end{abstract}

$(\Im$ Neurol Neurosurg Psychiatry 1992;55:806-810)

The alien hand sign, first described by Brion and Jedynak in 1972, referred to denial of hand ownership and the inability to transfer functions between the hemispheres in patients with corpus callosum tumours. ${ }^{1}$ Some patients thought that one hand felt foreign or alien; they could not imitate hand postures of one hand with the other and could not write. Other patients had tactile anomia and apraxia in response to verbal commands of the involved hand (verbal apraxia): they tended to confabulate responses to questions. When visual cues were removed (one hand held behind the back), these patients could not differentiate their own hand from the examiner's by feeling with their normal hand.

Since the initial report, several authors have described alien limbs in association with contralateral frontal strokes; ${ }^{2-4}$ corpus callosum infarction; $;^{5}$ anterior communicating artery rupture; ${ }^{6-9}$ corpus callosectomy; ${ }^{10}$ combination of a posterior corpus callosum lesion and contralateral thalamic sensory lesion; ${ }^{11}$ and bifrontal penetrating cerebral injury. ${ }^{8}$

More recently, alien limbs have been described in patients with proven and presumed corticobasal ganglionic degeneration (CBD). ${ }^{12-14}$ In addition to "alien-ness" and the other original features, patients often experienced involuntary complex motor activities. Observations include grasp reflexes and motor preservation, usually on the same side as the involuntary movements; hemiparesis and hemisensory changes; a wide range of involuntary complex motor behaviour, from grasping and groping to self destructive activity or intermanual conflict; and subtle speech and language abnormalities. A review of the various usages of alien hand and its evolving definition as a syndrome may be found in another publication. ${ }^{15}$

Despite numerous clinical descriptions, no systematic approach has been proposed for comprehensive examination of patients with the alien hand sign. The reports suggest that more than one anatomical lesion or pathological process accounts for alien limb phenomena, but no consistent anatomic correlates have emerged. We therefore studied patients with alien hand and related signs in order to suggest a comprehensive assessment and possible anatomical mechanisms.

\section{Methods}

We reviewed the hospital and clinic records of patients presenting to the neurology department with alien hand sign or related sensorimotor phenomena. The study period spanned from 1988 to the present. Out of the seven patients in the study, videotapes are available for five, all of whom had CBD. All patients had complete histories and physical examinations, neuroimaging studies and laboratory investigations to rule out metabolic disorders.

Patients were included if they complained of a foreign or alien limb, or carried a diagnosis of alien limb provided they also had complex, autonomous motor activity that was involuntary. Patients were excluded if they had apraxia or an identifiable movement disorder, for example, hemiballismus that fully explained the involuntary motor phenomenon (see appendix).

\section{Results}

Four patients were women and all were right handed except patient 7 who was ambidextrous. Age of onset ranged from 57 to 85 . Four 
Table 1 The alien hand and related signs, clinical features and progression

\begin{tabular}{|c|c|c|c|c|c|c|}
\hline Patient & Age of onset (years) & Sex & Aetiology & Handedness & Onset side & Progression \\
\hline $\begin{array}{l}1 \\
2 \\
3 \\
4 \\
5 \\
6 \\
7\end{array}$ & $\begin{array}{l}63 \\
60 \\
57 \\
72 \\
69 \\
85 \\
63\end{array}$ & $\begin{array}{l}F \\
F \\
F \\
M \\
M \\
F \\
M\end{array}$ & $\begin{array}{l}\text { CBD } \\
\text { CBD } \\
\text { CBD } \\
\text { CBD } \\
\text { CBD }+1-\text { infarction } \\
\text { infarction } \\
\text { infarction }\end{array}$ & $\begin{array}{l}\mathbf{R} \\
\mathbf{R} \\
\mathbf{R} \\
\mathbf{R} \\
\mathbf{R} \\
\mathbf{R} \\
\text { Mixed }\end{array}$ & $\begin{array}{l}\text { L UE } \\
\text { L UE } \\
R \text { UE } \\
\text { R UE } \\
\text { R UE } \\
\text { R UE } \\
\text { Both UEs }\end{array}$ & $\begin{array}{l}\text { Wihin } 2 \text { years, LLE \& RLE } \\
\text { Within } 2 \text { years, RUE } \\
\text { Within } 2 \text { years, LUE \& RLE } \\
\text { Within } 4 \text { years RLE } \\
\text { Within } 4 \text { years, RLE \& LUE } \\
\text { Within days, RLE } \\
\text { Resolved }\end{array}$ \\
\hline
\end{tabular}

patients had CBD and two had multiple strokes (table 1). Patient 5 had a presentation and course similar to what has been described for CBD, but also had multiple lesions on CT and a left slow-wave focus on EEG, raising the possibility of multiple infarcts alone or in combination with CBD. His symptoms, however, predated his CT findings (he had an earlier, normal study), and were slowly progressive, which makes a stroke related aetiology less likely.

The alien limb initially involved a hand in all seven patients, and progressed to involve other limbs in six of the seven (table 1). Patient 7, who was ambidextrous, had a bilateral onset. His condition resolved within a few days of an acute right frontal infarct, but the alien limbs persisted in all other patients until lost to follow up or until the limb became stiff and useless. In the CBD patients, the illness either began with development of an alien hand or with Parkinsonism, later complicated by an alien hand sign. The two patients with cerebral infarcts varied: one had the sudden onset of alien hand and a right parietal syndrome, while the other had the insidious onset of intermanual conflict. For example, he noted that one hand tried to turn left when the other hand tried to turn right while driving a car.

All patients complained, at some time in their illness, of involuntary complex motor activity and the perception that their limb either did not obey them or that it did not belong to them. All had difficulty carrying out verbal commands with the affected limb(s). Six of the seven patients displayed some non-goal directed motor activity, such as posturing, grasping and groping, or action induced patterned, rhythmical movements (table 2). Two patients had self destructive behaviours which were autonomous but purposeful, in addition to the non-goal directed behaviours. Four of seven, all with CBD, had cortical reflex myoclonus. At the time of examination, four patients had difficulty with bimanual coordination, on tasks such as alternating fist closure and opening or taking off glasses, folding the frame and putting them away. Grasp reflex was common (five patients) and occurred with both causes. Abnormalities of the ability to alternate motor sets (fist-ring task or fist-palmside, ${ }^{16}$ somaesthetic transfer (cross-localising fingertip stimulation), and other complex sensory functions (graphesthesia, stereognosis) were frequently noted. Patients 1,3 and 5 had slow, paraphasic speech and patient 2 had dysnomia. None showed the transcortical motor aphasia sometimes described in association with supplementary motor area lesions. ${ }^{17}$
We excluded one patient who had several elements of the alien hand sign but did not meet the full criteria which we proposed. This presumed CBD patient presented four years into an illness which began with speech apraxia and right sided clumsiness. When examined, she was completely mute, had trouble with somaesthetic transfer (cross replicating hand postures) and bimanual coordination. She frequently held her right arm with her left hand. When asked to touch her nose, she rubbed the side of her head or her face before ultimately touching the nose. Comprehension for three step commands not involving the right limbs was good. We suspect that alien limb could have been diagnosed earlier in her disease, when she could have told us whether or not her limb activity felt foreign or alien.

\section{Discussion}

The term alien hand has been used to describe a wide variety of sensory impressions and motor behaviours. Minimal diagnostic criteria have not been set. We define alien limb as a feeling that one limb is foreign or "has a will of its own", together with observable involuntary motor activity. Our study and review of published reports support this definition for all cases of alien hand, regardless of cause. The feeling of alien-ness can range from a perception that the limb belongs to the examiner rather than the patient to personification of the limb. Such personifications can be quite elaborate and patients might be mistakenly thought psychotic: patient 6 believed that her left arm was a baby called Joseph, and that its actions against other parts of her body (like pinching her nipples) were mischievous behaviours (biting while nursing). The autonomous motor activities seen constitute a spectrum of complexity from action induced patterned and rhythmical movement to non-goal directed grasping and groping behaviour, goal directed activities like utilisation behaviour (the compulsive manipulations of tools) or even self destructive acts. Problems with bimanual coordination are always present at some point in the history. These bimanual coordination problems include mirror movements, where one hand involuntarily mimics the other, and conflicts between the hands, such as a struggle between the hands as each attempts to answer the telephone (patient 7). The term "diagnostic dyspraxia" has been used to refer to abnormal involuntary hand movements elicited by voluntary movement of the other hand, as opposed to non-triggered involuntary movements. ' Use of this term is misleading 
Table 2 Presentation, signs and symptoms * of alien hand

\begin{tabular}{|c|c|c|c|}
\hline Patient & Aetiology & Onset type & Major features \\
\hline 1 & CBD & $\begin{array}{l}\text { Parkinsonism } \\
\text { (gait and speech) } \\
\text { L alien hand }\end{array}$ & $\begin{array}{l}\mathrm{L} \text { grasp and reflex myoclonus } \\
\mathrm{L} \text { rigidity and action tremour } \\
\text { Posturing, mirror movements }\end{array}$ \\
\hline 2 & CBD & $\mathrm{L}$ alien hand & $\begin{array}{l}\text { Bilateral reflex myoclonus } \\
\text { L rigidity and posturing } \\
\text { Some goal directed activity }\end{array}$ \\
\hline 3 & CBD & $\mathbf{R}$ alien hand & $\begin{array}{l}R \text { grasp and reflex myoclonus } \\
R \text { rigidity and posturing }\end{array}$ \\
\hline 4 & CBD & $\mathrm{R}$ alien hand & $\begin{array}{l}\mathbf{R} \text { grasp and rigidity } \\
\text { Bilateral action tremour }\end{array}$ \\
\hline 5 & CBD $+/-$ infarction & $\begin{array}{l}\mathbf{R} \text { alien hand and Parkinsonism } \\
\text { with gait and speech }\end{array}$ & $\begin{array}{l}\mathrm{R} \text { graps and rigidity } \\
\text { Bilateral reflex myoclonus } \\
\text { Bilateral action tremours }\end{array}$ \\
\hline 6 & Infarction & $\mathrm{L}$ alien hand & $\begin{array}{l}\text { Goal directed, self destructive and posturing } \\
\text { L hemi-inattention }\end{array}$ \\
\hline 7 & Infarction & Intermanual conflict & $\begin{array}{l}\mathrm{R} \text { grasp } \\
\text { Motor perseveration }\end{array}$ \\
\hline
\end{tabular}

CBD = Corticobasal ganglionic degeneration; $L=$ left; $R=$ right.

*All patients displayed verbal apraxia with the affected limb(s) and had difficulty with bimanual coordination by history or on examination.

and our data suggest that so called triggered and non-triggered movements can occur in the same patient.

Other clinical features should be sought which are frequently associated with the alien limb. They may provide clues to anatomy and cause of the alien hand, as well as aid in differential diagnosis. Ultimately, associated features may give rise to syndromes with particular aetiologies or neuropathologic lesions, although reports have not been complete enough to date. Associated features include frontal lobe signs such as a grasp reflex or difficulty shifting motor sets, cortical reflex myoclonus, particularly in CBD patients, and other historical and examination features (appendix).

The differential diagnosis of alien limb includes grasp reflex, usually secondary to frontal lobe pathology, athetosis and pseudoathetosis, action dystonia, hemiballismus, and hemiataxia. In these disorders, the limb does not feel foreign and the patient does not deny ownership of the affected limb. Manoeuvres such as placing both hands behind the patient's back and then asking him or her to differentiate the alien limb from the examiner's limb may be useful in these conditions. Neglect syndromes secondary to parietal disease or optic ataxia secondary to occipital pathology may induce a sense of foreignness or the feeling that a limb does not "follow commands". These patients do not, however, have autonomous involuntary motor activities.

We have also observed elements of alien limb in patients with Alzheimer's disease (AD). An unsuppressed grasp reflex is sometimes observed, for example on finger-nose-finger testing, and may involve one limb more than the other. $\mathrm{AD}$ patients occasionally display other involuntary behaviours, similar to utilisation behaviour, such as compulsively grasping writing utensils and scribbling with them, or compulsively picking up documents and turning the pages. These patients, because of their global cognitive deficits, may have difficulty with somaesthetic transfer, verbal praxis, and bimanual coordination tasks similar to patients with alien hand. Unlike patients with alien hand, the findings in $A D$ patients are frequently subtle, fragmentary and transient.
Our study adds new information to the anatomical causes described in alien hand. Unilateral mesial frontal lesions which affect the anterior cingulate gyrus and supplementary motor areas (SMA) can reportedly cause contralateral alien hand sign, but corpus callosum involvement could not be ruled out in these cases. ${ }^{34}$ Anterior corpus callosum resection alone can result in a transient alien hand on the non-dominant side. ${ }^{10}$ Bifrontal lesions affecting the SMA and corpus callosum have been associated with the syndrome, although diagnostic criteria varied. ${ }^{5-8}$ Our patient 7, with bilateral subcortical lesions adjacent to the anterior corpus callosum differs from these reports (see table 3). His symptoms were bilateral at the onset, possibly because he was ambidextrous or because he had bilateral infarcts. Unlike other cases his lesions did not directly involve the corpus callosum or SMA, but possibly disconnected each SMA from the opposite side. The intermanual conflicts present at onset improved following a right frontal infarct, suggesting that additional disruption of the SMA or related structures on the right "corrected" the initial imbalance.

One patient who displayed phenomena which meet our criteria for the alien hand resulting from a combination of crossed optic ataxia (right parieto-occipital lesion involving corpus callosum) and contralateral sensory ataxia (right thalamic lesion) has been reported on. " Our patient 6 also had a large right posterior infarct that affected the corpus callosum and a right internal capsule lacune. She had similar behavioural symptomatology, including coarse movements of the alien limb, the belief that the affected hand had hostile motivations, and eventual adjustment to the "alien" presence by treating it like her child. Our patient did not have a thalamic sensory lesion, which was thought to be necesary to explain the alien hand sign in the other case. Perhaps involvement of sensory fibres in the internal capsule in our patient was analogous to the other patient's thalamic lesion; alternatively, a large posterior infarct alone may be sufficient to give an alien hand sign, although this has never been reported.

A recent report suggests two distinct alien hand syndromes (AHS): a frontal type (frontal 
Table 3 Focal imaging abnormalities in patients with alien hand sign

\begin{tabular}{ll}
\hline Patient & Focal findings \\
\hline 5 & $\begin{array}{l}\text { Small } R \text { occipital infarct, adjacent to the posterior } \\
\text { horn. }\end{array}$ \\
$\begin{array}{l}\text { Small } R \text { anterior temporal infarct. ( } L \text { slow wave } \\
\text { focus on EEG) }\end{array}$ \\
$\begin{array}{l}\mathrm{R} \text { infarct of posterior temporo-occipital lobe also } \\
\text { involving posterior parietal lobe and posterior limb } \\
\text { of internal capsule. } R \text { basal ganglia, } L \text { caudate, and } \\
\text { L cerebellar infarcts. }\end{array}$ \\
$\begin{array}{l}\mathbf{R} \text { and } L \text { subcortical white matter (adjacent to } \\
\text { corpus callosum), } R \text { putamen, and vague } R \text { parietal } \\
\text { and L parietotemporal ischaemic regions. New } R \\
\text { frontal infarct (luxury perfusion) and R parietal and }\end{array}$ \\
L parietotemporal perfusion defects by SPECT.
\end{tabular}

$\bar{L}=$ left; $R=$ right; SPECT $=$ single proton emission computerised tomography.

AHS) associated with reflexive grasping, groping and the compulsive manipulation of tools and a callosal type (callosal AHS) characterised by intermanual conflict and the absence of frontal features. ${ }^{18}$ Frontal AHS usually involves the dominant hand and is due to dominant SMA, anterior cingulate gyrus, medial pre-frontal cortex and anterior corpus callosum damage. Callosal AHS involves the non-dominant hand and is caused by isolated corpus damage. ${ }^{18}$ This classification is incomplete and may be misleading for several reasons. First, the cases drawn from reports to illustrate callosal AHS included patients with signs and symptoms of more extensive lesions. Watson and Heilman's patient had transcortical motor aphasia and hypokinesia, as well as signs of collosal disconnection, due to angiographically demonstrated anterior cerebral artery spasm. ${ }^{5}$ Although her frontal symptoms resolved, and CT scan (five months later) showed only a callosal infarct, the authors could not exclude the possibility of frontal lobe damage. Similarly, two other cases classified as callosal AHS showed clinical features indicative of frontal dysfunction. ${ }^{7}$

Feinberg et al suggest that patients with callosal AHS do not have frontal signs, such as "reflex grasping". ${ }^{18}$ Yet several of their patients had grasp reflexes, as did our patient 7 with bifrontal lesions, which in their review always resembled callosal AHS. Grasp reflex is not commonly attributed to callosal section alone, but is viewed as a "neighbouring sign" of frontal lobe injury. ${ }^{10}$ The classification system of frontal versus callosal AHS does not account for Levine and Rinn's patient ${ }^{11}$ or our patient 6 who had non-frontal lesions of the nondominant hemisphere. The anatomical speculations which underlay this classification cannot explain alien limbs in patients with CBD, which the majority of our patients had. Yet the phenomenology of alien hand in CBD overlaps with AHS due to other aetiologies. Finally, the proposed definitions for frontal and callosal AHS mention "uncontrollable movements of an extremity not due to a movement disorder", but do not include the feeling of foreign-ness or alien-ness which has been a hallmark of the AHS.

There have been few patients with alien limb and pathologically proven CBD. ${ }^{13}$ In one such patient (case 3) clinical details are sketchy but the postmortem examination showed cell loss in the substantia nigra, locus ceruleus and nucleus ambiguous with corticobasal inclusion bodies, and little involvement of cortex. ${ }^{13}$ Postmortem examinations in two other patients showed changes characteristic of CBD most prominent in the left medial frontal region, ipsilateral to the side of initial and greater clinical involvement in one patient and changes in the central frontoparietal cortex, most prominent contralateral to the side of onset in another. ${ }^{14}$ The substantia nigra was severely affected in both cases. Spontaneous levitation and posturing, as seen in our patients $1,2,3$, and 6 (table 2) may be more common in CBD, and the previously published reports are consistent with this hypothesis.

Unilateral lesions of the SMA in subhuman primates cause abnormal bimanual coordination, seemingly because the remaining SMA induces similar movements in both hands simultaneously. ${ }^{19}$ This bimanual coordination deficit occurs without involuntary complex motor activity and is abolished by subsequent complete callosal section. There is also some variability in clinical presentation depending on whether the SMA lesion occurs ipsilateral or contralateral to the dominant hand. ${ }^{19}$ If the SMA lesion is on the non-dominant side, the non-dominant hand may actually improve some motor skills as it comes to resemble the dominant hand, presumably because both sides are now modulated by the dominant SMA. In our limited number of patients, we could not discern a clinical pattern related to handedness or side of onset.

The primate findings suggest that unilateral lesions of the SMA may account for some of the bimanual coordination problems which were universal in our patients, but that unilateral SMA injury, disconnection, or degeneration alone does not likely produce all of the features of alien hand. Additional lesions of the corpus callosum or bilateral involvement of fibres from the SMA may be necessary in order to manifest the autonomous motor activity and sensation of alien-ness. The observation that surgically induced callosal section produces transient alien hand also supports the theory that both SMA and corpus callosum must be affected to produce a persistent sign in those patients with frontal pathology. Lesions involving central sensory nuclei or tracts in combination with posterior parieto-occipital and corpus callosum lesions may give a picture similar to alien hand, but by different mechanisms. Such patients lose visual guidance of the affected limb (optic ataxia) as well as sensory input from that limb. In the setting of neglect syndrome affecting the hand, these combined deficits could give phenomonology consistent with alien hand (alien-ness and seemingly autonomous motor activity). These patients with posterior lesions also tended to personify their alien limbs, which we did not see in other cases. Basal ganglia dysfunction due to infarcts or degenerative processes may intensify features of the alien hand and modify the related signs.

The alien hand can be quite disabling to patients as well as psychologically disturbing. 
Although no specific therapy exists for the alien hand sign, experimental neurosurgical approaches to modulating corpus callosum or supplementary motor area activity remain a possibility, particularly in light of data from subhuman primate studies.

Presented in part at the American Academy of Neurology, 23 April 1991, Boston, USA.

Dr Doody was supported, in part, by an ADRC grant NIH USPH-I-P50-AG08664 and by a grant from the Hershel and Hilda Rich Philanthropic Fund.

1 Brion S, Jedynak CP. Troubles du transfert interhemispherique. Revue Neurol (Paris) 1972;126(4):257-66.

2 Shahani B, Burrows P, Whitty WM. The grasp reflex and perseveration. Brain 1970;93:181-92.

3 Goldberg G, Mayer MH, Toglia JU. Medial frontal cortex infarction and the alien hand sign. Arch Neurol infarction and

4 McNabb AW, Carroll WM, Mastaglia FL. "Alien hand" and loss of bimanual coordination after dominant anterior cerebral artery territory infarction. F Neurol Neurosurg Psychiatry 1988;51:218-22.

5 Watson RT, Heilman KM. Callosal apraxia. Brain 1983;106:391-403.

6 Starkstein SE, Berthier M, Leiguarda R. Disconnection syndrome is a right-handed patient with right hemispheric speech dominance. Eur Neurol 1988;28:187-90.

7 Leiguarda R, Starkstein S, Berthier M. Anterior callosal hemorrhage. Brain 1989;112:1019-37.

8 Banks G, Short P, Martinez AJ, Latchaw R, Ratcliff G, Boller F. The alien hand syndrome. Arch Neurol 1989;46:456-9.

9 Tanaka Y, Iwasa H, Yoshida M. Diagnostic dyspraxia: case report and movement-related potentials. Neurology 1990;40:657-61.

10 Bogen JE. The callosal syndrome. In: Heilman KM, Valenstein E, eds. Clinical Neuropsychology, 2nd ed, New York: Oxford Univ Press, 1985:295-338.

11 Levine DN, Rinn WE. Opticosensory ataxia and alien hand syndrome after posterior cerebral artery territory infarction. Neurology 1986;36:1094-7.

12 Jankovic J. The Relationship between Parkinson's disease and other movement disorders. In: Calne DB, ed. Handbook of Experimental Pharmacology. Berlin: SpringerVerlag, 1989:227-70.

13 Gibb WRG, Luther PJ, Marsden CD. Corticobasal degeneration. Brain 1989;112:1171-92.

14 Riley DE, Lang AE, Lewis A, Resch L, Ashby P, Hornykiewicz O, Black S. Cortical-basal ganglionic degeneration. Neurol 1990;40:1203-12.

15 Doody RS. The alien hand. In: Molgaard CA, ed. Neuroepidemiology: theory and method. Orlando: Academic Press, in press.

16 Strub RL, Black FW. The mental status examination in neurology, 2nd ed. Philadelphia: F A Davis, 1985

17 Alexander MP, Benson DF, Stuss DT. Frontal lobes and language. Brain and Lang 1989;37:656-91.

18 Feinberg TE, Schindler RJ, Flanagan NG, Haber LD. Two alien hand syndromes. Neurology, in press.

19 Brinkman C. Supplementary motor area of the monkey's cerebral cortex: short- and long-term deficits after unilateral ablation and the effects of subsequent callosal section. $\mathcal{f}$ Neuroscience 1984;4(4):918-29.

Appendix

Evaluation of alien hand and related signs

A Historical information

1 Aetiology?
2 Did alien hand develop early or late in the illness?

3 Which limbs were involved, and in what sequence?

4 How many years until other limbs involved?

5 Age at onset?

6 Relationship to hand dominance?

7 Was alien hand on the same side as the other pathology or not?

8 Were the following phenomena present, and when in the course of the illness?

a persistent grasp;

b denial of ownership and/or autocriticism (patient critical of limb's actions) or personification (attributes a personality to the limb);

c autonomous complex motor activity;

d was the activity non-goal directed (grasping and groping);

e was it goal directed (compulsive utilisation behaviour; self destructive behaviour);

f problems with bimanual coordination;

$\mathrm{g}$ intermanual conflicts (competing hand movements not triggered by the unaffected hand) or diagnostic dyspraxia (competing hand movements triggered by moving the unaffected hand), or mirror movements (one hand mirrors the other involuntarily).

\section{B Examination}

1 Grasp reflex.

2 Cortical reflex myoclonus.

3 Alternating motor sets (fist-ring alternations, fist-palm-side, etc).

4 Complex sensory function (graphesthesia, stereognosis)

5 Tactile naming.

6 Somaesthetic transfer (cross replicating hand postures, cross localising fingertip stimulation).

7 Ownership of arm held behind back with examiner's arm as foil.

8 Sequential unilateral movements (squeeze and then flex, or touch each finger to the thumb in order).

9 Bimanual coordination (buttoning, taking something out of a container, opening and closing hands alternately).

10 Observation for mirror movements, or intermanual conflicts.

11 Praxis to verbal command, followed by mimesis and use of an object.

12 Language (initiative, spontaneous speech, repetition, comprehension, paraphasias, writing). 